\title{
Thermal Conductivity of Ordered Mesoporous Nanocrystalline Silicon Thin Films Made from Magnesium Reduction of Polymer- Templated Silica
}

\author{
Jin Fang, ${ }^{\dagger}$ Chris B. Kang, ${ }^{\ddagger}$ Yi Huang, ${ }^{\dagger}$ Sarah H. Tolbert, ${ }^{*} \stackrel{\dagger}{\ddagger}$ and Laurent Pilon $*^{\dagger}$ \\ ${ }^{\dagger}$ Henry Samueli School of Engineering and Applied Science, Mechanical and Aerospace Engineering Department, University of \\ California, Los Angeles, California 90095-1597, United States \\ ${ }^{\ddagger}$ Department of Chemistry and Biochemistry, University of California, Los Angeles, California 90095-1569, United States
}

\begin{abstract}
This paper reports the cross-plane thermal conductivity of ordered mesoporous nanocrystalline silicon thin films between 25 and $315 \mathrm{~K}$. The films were produced by evaporation-induced self-assembly of mesoporous silica followed by magnesium reduction. The periodic ordering of pores in mesoporous silicon was characterized by X-ray diffraction and direct SEM imaging. The average crystallite size, porosity, and film thickness were about $13 \mathrm{~nm}, 25-35 \%$, and $140-340 \mathrm{~nm}$, respectively. The pores were arranged in a

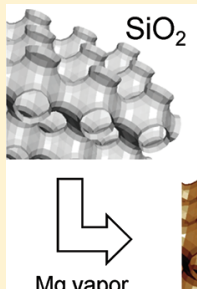

Mg vapor

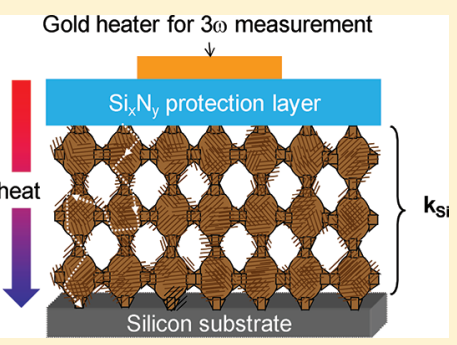
face-centered cubic lattice. The cross-plane thermal conductivity of the mesoporous silicon thin films was measured using the $3 \omega$ method. It was between 3 and 5 orders of magnitude smaller than that of bulk single crystal silicon in the temperature range considered. The effects of temperature, film thickness, and copolymer template on the thermal conductivity were investigated. A model based on kinetic theory was used to accurately predict the measured thermal conductivity for all temperatures. On one hand, both the measured thermal conductivity and the model predictions showed a temperature dependence of $k \propto T^{2}$ at low temperatures, typical of amorphous and strongly disordered materials. On the other hand, at high temperatures the thermal conductivity of mesoporous silicon films reached a maximum, indicating a crystalline-like behavior. These results will be useful in designing mesoporous silicon with desired thermal conductivity by tuning its morphology for various applications.
\end{abstract}

\section{INTRODUCTION}

Porous silicon has been extensively investigated due to its wide range of applications. It has been used in optoelectronics for its photoluminescence properties. ${ }^{1}$ It has also been used as a thermal insulator and sensor in Micro-Electro-Mechanical Systems (MEMS) due to its low thermal conductivity and rigid solid structure. ${ }^{2}$ In addition, nanoporous silicon is also promising in highly energetic MEMS devices. ${ }^{3-5}$ When exposed to both oxygen and heat, strong exothermic reactions take place within the nanostructures. This can be used for microthrusters, microinitiators, and gas generation for actuators. ${ }^{5}$ More recently, nanostructured silicon was identified as a thermoelectric material with an operating temperature ranging from 573 to $1273 \mathrm{~K}$ for energy-harvesting purposes. ${ }^{6-8}$ Its low thermal conductivity $k$, high electrical conductivity $\sigma$, and high Seebeck coefficient $S$ contribute to a high thermoelectric figure of merit $Z T$ defined as $Z T=\sigma S^{2} T / k$ at temperature $T$. In all these applications, knowledge of the thermal properties of porous silicon over a wide range of temperatures is of significant importance for its practical implementation in devices.

\section{BACKGROUND}

2.1. Measurements. Drost et al. ${ }^{9}$ reported the thermal conductivity of nanoporous silicon made by electrochemical etching of bulk single crystal silicon. The film thickness and porosity were $10 \mu \mathrm{m}$ and $40-53 \%$, respectively. However, pore size was not specified. The measured thermal conductivity was 1.2 and $1.75 \mathrm{~W} / \mathrm{m} \cdot \mathrm{K}$ for p-type and n-type nanoporous silicon at room temperature, respectively. Several other experimental studies reported the thermal conductivity of electrochemically etched porous silicon films measured using the photoacoustic method. ${ }^{10-13}$ The film thickness and the porosity ranged from tens to hundreds of micrometers and from $20 \%$ to $75 \%$, respectively. The pores were usually cylindrical and perpendicular to the film substrate, with diameter ranging from tens to hundreds of nanometers. ${ }^{10-13}$ In addition, Périchon et al. ${ }^{14}$ reported the thermal conductivity of $50 \mu \mathrm{m}$ thick porous silicon film with $50 \%$ porosity measured using the micro-Raman scattering method at room temperature. However, the pore size was not reported. Wolf and Brendel ${ }^{15}$ measured the in-plane thermal conductivity of porous silicon free-standing films using

Received: March 16, 2012

Revised: May 16, 2012

Published: May 16, 2012 
a lock-in thermography technique. The sample thickness, porosity, and pore size were $3-27 \mu \mathrm{m}, 27-66 \%$, and about 0.1 $\mu \mathrm{m}$, respectively. ${ }^{15}$ Moreover, Gomès et al. ${ }^{16}$ measured the thermal conductivity of electrochemically etched mesoporous silicon thin films with thickness ranging from $38 \mathrm{~nm}$ to $7.2 \mu \mathrm{m}$, porosity from $30 \%$ to $80 \%$, and crystallite size from 10 to 20 $\mathrm{nm}$ using scanning thermal microscopy. ${ }^{17,18}$ Note that, in all previously mentioned studies, the thermal conductivity was measured at room temperature and was about 2 orders of magnitude smaller than that of bulk single crystal silicon equal to $148 \mathrm{~W} / \mathrm{m} \cdot \mathrm{K}$. $^{19}$

In terms of temperature-dependent studies of porous silicon, Gesele et al. ${ }^{20}$ measured the cross-plane thermal conductivity of electrochemically etched porous silicon films between 35 and $315 \mathrm{~K}$ using the $3 \omega$ method. The film thickness, porosity, and crystallite size were about $21-46 \mu \mathrm{m}, 64-89 \%$, and $1.7-9 \mathrm{~nm}$, respectively. ${ }^{20}$ The measured thermal conductivity typically ranged from 0.01 to $0.8 \mathrm{~W} / \mathrm{m} \cdot \mathrm{K}$ and increased with increasing temperature. ${ }^{20}$ Song and Chen ${ }^{21}$ measured the in-plane thermal conductivity of macroporous silicon films from 50 to $300 \mathrm{~K}$. The through-film cylindrical pores were periodically arranged and fabricated using photolithography. The film thickness, porosity, and pore size varied from 4 to $7 \mu \mathrm{m}, 17 \%$ to $26 \%$, and 2 to $10 \mu \mathrm{m}$, respectively. More recently, Hopkins et al. ${ }^{22}$ reported the room-temperature cross-plane thermal conductivity of $500 \mathrm{~nm}$ thick silicon films with etched through-film cylindrical pores arranged in a two-dimensional simple cubic lattice. The pore diameter and porosity varied from 300 to 400 $\mathrm{nm}$ and from $20 \%$ to $28 \%$, respectively. The reduction in thermal conductivity was attributed to phonon boundary scattering as well as phonon confinement effect. ${ }^{22}$

Finally, Wang et al. $^{23}$ reported the cross-plane thermal conductivity of nanocrystalline silicon films about $1 \mathrm{~mm}$ thick prepared using a current activated and pressure-assisted densification technique. The porosity and the average grain size varied from 0 to $17 \%$ and from 64 to $550 \mathrm{~nm}$, respectively. The measured thermal conductivity showed a temperature dependence of $k \propto T^{2}$ at low temperatures. The author developed a frequency-dependent relaxation time model for phonon-grain boundary scattering to accurately predict the thermal conductivity of nanocrystalline silicon over a wide range of temperatures.

Most porous silicon films investigated in previous studies were made by electrochemical etching with cylindrical pores and branches. Their sizes were usually on the order of a few to tens of micrometers. ${ }^{24}$ Non-close-packed crystalline silicon colloidal nanostructures have also been synthesized using a laser-induced transient melt process, and porous silicon in submicrometer scale can be produced. ${ }^{25}$ Recently, ordered mesoporous silicon films with closely packed pores were produced from the mesoporous silica framework using a combination of evaporation-induced self-assembly and magnesium reduction. ${ }^{26}$ This method enables one to tune the porosity as well as the pore size and spatial arrangement of the mesoporous materials and, in turn, their thermophysical properties. $^{26}$

2.2. Modeling. Kinetic Theory Model. On the basis of kinetic theory and relaxation time approximation, the thermal conductivity of crystalline materials is expressed as ${ }^{27}$

$$
k_{\mathrm{m}}(T)=\frac{1}{3} \sum_{i=1}^{3} \int_{q} \hbar \omega_{i}(q) D_{i}(q) \frac{\partial f_{\mathrm{BE}, i}(q)}{\partial T} v_{\mathrm{g}, i}^{2}(q) \tau_{\mathrm{tot}, i}(q) \mathrm{d} q
$$

where $k_{\mathrm{m}}$ is the solid matrix thermal conductivity; subscript $i$ represents the longitudinal or the transverse polarizations; $q$ is the wavevector; $\omega_{i}(q)$ is the angular frequency; $\hbar=1.054 \times$ $10^{-34} \mathrm{~m}^{2} \mathrm{~kg} / \mathrm{s}$ is the reduced Planck's constant; $D_{i}(q)=q^{2} / 2 \pi^{2}$ is the phonon density of states; $f_{\mathrm{BE}, i}(q)$ is the Bose-Einstein distribution given by $f_{\mathrm{BE}, i}(q)=1 /\left[\exp \left(\hbar \omega_{i}(q) / k_{\mathrm{B}} T\right)-1\right]$ where $k_{\mathrm{B}}=1.38 \times 10^{-23} \mathrm{~J} / \mathrm{K}$ is the Boltzmann constant; $v_{\mathrm{g}, i}=$ $d \omega_{i}(q) / d q$ is the phonon group velocity; and $\tau_{\text {tot }, i}(q)$ is the total phonon scattering relaxation time. ${ }^{27}$ Note that the summation is over one longitudinal $(i=1)$ and two transverse polarizations $(i=2,3)$.

A fourth-order polynomial dispersion relation for silicon was suggested by Hopkins et al. ${ }^{28}$ and given by

$$
\omega_{i}(q)=a_{i} q+b_{i} q^{2}+c_{i} q^{3}+d_{i} q^{4}
$$

The authors determined the coefficients $a_{i}, b_{i}, c_{i}$, and $d_{i}$ by fitting eq 2 to the measured dispersion relation for bulk silicon in the [100]-direction. ${ }^{29}$ For the longitudinal polarization, they were reported to be $a_{1}=8350 \mathrm{~m} / \mathrm{s}, b_{1}=2.94 \times 10^{-8} \mathrm{~m}^{2} / \mathrm{s}, c_{1}=$ $-3.53 \times 10^{-17} \mathrm{~m}^{3} / \mathrm{s}$, and $d_{1}=1.37 \times 10^{-27} \mathrm{~m}^{4} / \mathrm{s}$, while for the transverse polarizations, they were $a_{2}=a_{3}=6090 \mathrm{~m} / \mathrm{s}, b_{2}=b_{3}$ $=-1.86 \times 10^{-7} \mathrm{~m}^{2} / \mathrm{s}, c_{2}=c_{3}=-3.36 \times 10^{-17} \mathrm{~m}^{3} / \mathrm{s}$, and $d_{2}=d_{3}$ $=1.94 \times 10^{-27} \mathrm{~m}^{4} / \mathrm{s}^{28}$ Integration over $q$ is typically truncated up to a cutoff wavenumber $q_{\max }=1.2 \times 10^{10} \mathrm{~m}^{-1} .^{28}$ This corresponds to cutoff frequencies $\omega_{\max , 1}=71.6 \mathrm{Trad} / \mathrm{s}$ and $\omega_{\max , 2}=\omega_{\max , 3}=28.3 \mathrm{Trad} / \mathrm{s}$.

For bulk crystalline silicon, the total phonon relaxation time $\tau_{\text {bulk }, i}$ for polarization $i$ can be related to the relaxation time for Umklapp scattering $\tau_{\mathrm{U}, i}$, defect scattering $\tau_{\mathrm{D}, i}$, and boundary scattering $\tau_{\mathrm{B}, i}$ via the Matthiessen rule as ${ }^{27}$

$$
\frac{1}{\tau_{\mathrm{bulk}, i}}=\frac{1}{\tau_{\mathrm{U}, i}}+\frac{1}{\tau_{\mathrm{D}, i}}+\frac{1}{\tau_{\mathrm{B}, i}}
$$

where the relaxation times $\tau_{\mathrm{U}, i}, \tau_{\mathrm{D}, i}$ and $\tau_{\mathrm{B}, i}$ are given by ${ }^{30,28}$

$$
\begin{aligned}
& 1 / \tau_{\mathrm{U}, i}=B T \omega_{i}^{2}(q) \exp (-C / T), \quad 1 / \tau_{\mathrm{D}, i}=D \omega_{i}^{4}(q), \\
& \quad \text { and } 1 / \tau_{\mathrm{B}, i}=v_{\mathrm{g}, i}(q) / E
\end{aligned}
$$

Here, the coefficients $B, C, D$, and $E$ are fitting parameters. Furthermore, for nanocrystalline silicon, additional phonon scattering by crystallite grain boundaries becomes important. Then, the total relaxation time for nanocrystalline silicon $\tau_{\mathrm{NC}, i}$ can be simply expressed as

$$
\frac{1}{\tau_{\mathrm{NC}, i}}=\frac{1}{\tau_{\mathrm{bulk}, i}}+\frac{1}{\tau_{\text {grain }, i}}
$$

where $\tau_{\text {grain }, i}$ is the relaxation time for phonon scattering by crystallite grain boundaries.

A commonly used model suggests that $\tau_{\text {grain, } i}$ can be expressed as $1 / \tau_{\text {grain }, i}=v_{\mathrm{g}, i} / d_{\text {cryst }}$ where $d_{\text {cryst }}$ is the crystallite size. $^{27}$ This model is based on two assumptions: (i) the effective phonon mean free path is limited by the crystallite size and (ii) phonon scattering by grain boundaries is frequency independent or "gray". ${ }^{23}$ However, Wang et al. ${ }^{23}$ recently indicated that a frequency-dependent model was required to accurately predict the thermal conductivity of nanocrystalline silicon. This model was given by ${ }^{23}$ 


$$
\frac{1}{\tau_{\text {grain }, i}}=\frac{v_{\mathrm{g}, i}}{\alpha d_{\text {cryst }}\left(0.7 \omega_{\max , i} / \omega_{i}\right)}
$$

where $\alpha$ is a fitting parameter accounting for the effect of grain boundary transmission and varies between 0 and 1 . The smaller value of $\alpha$ corresponds to lower phonon transmission through grain boundaries resulting in smaller thermal conductivity. ${ }^{23}$

Finally, the effect of porosity on thermal conductivity of mesoporous silicon should be accounted for by using some effective medium approximations (EMAs). ${ }^{28,31}$ For example, the coherent potential model gives the effective thermal conductivity $k_{\text {eff }}$ as ${ }^{32,33}$

$$
k_{\text {eff }}=k_{\mathrm{m}} \Psi_{\mathrm{cp}}\left(f_{\mathrm{v}}\right)=k_{\mathrm{m}}\left(1-1.5 f_{\mathrm{v}}\right)
$$

where $f_{\mathrm{v}}$ and $k_{\mathrm{m}}$ are the porosity and thermal conductivity of the solid matrix, respectively. This model was first derived by Landauer $^{32}$ for the effective dielectric properties of random mixtures of spherical inclusions in a continuous matrix. Cahill and Allen $^{33}$ successfully applied this model to predict the thermal conductivity of Vycor glass (amorphous) from 30 to $300 \mathrm{~K}$ with pore diameter and porosity approximately equal to $10 \mathrm{~nm}$ and $30 \%$, respectively. In addition, this model was also found to agree well with thermal conductivity predictions for amorphous nanoporous silica at $300 \mathrm{~K}$ obtained from nonequilibrium molecular dynamics simulations. ${ }^{34}$ More recently, a scaling law based on kinetic theory and the coherent potential EMA was found to accurately model the thermal conductivity of nanoporous crystalline silicon calculated using both equilibrium and nonequilibrium molecular dynamics simulations. $^{31,35}$ Note that in these latter studies the matrix was modeled as monocrystalline, and phonon scattering by grain boundaries was ignored.

Minimum Thermal Conductivity Model. As an alternative to the kinetic theory model [eqs 1-7], the minimum thermal conductivity of strongly disordered material $k_{\mathrm{m} \text {,min }}$ can be estimated using the following model derived by Cahill and Pohl $^{36}$

$$
k_{\mathrm{m}, \min }(T)=\left(\frac{\pi}{6}\right)^{1 / 3} k_{\mathrm{B}} n^{2 / 3} \sum_{i} v_{\mathrm{g}, i}\left(\frac{T}{\theta_{i}}\right)^{2} \int_{0}^{\theta_{i} / T} \frac{x^{3} e^{x}}{\left(e^{x}-1\right)^{2}} \mathrm{~d} x
$$

where $n=5.02 \times 10^{28} \mathrm{~m}^{-3}$ is the atomic number density of crystalline silicon; and $\theta_{i}$ is a characteristic temperature expressed as $\theta_{i}=v_{\mathrm{g}, i}\left(\hbar / k_{\mathrm{B}}\right)\left(6 \pi^{2} n\right)^{1 / 3} \cdot{ }^{36}$ This model has been termed the minimum thermal conductivity model and applies to amorphous and strongly disordered polycrystalline materials. $^{27}$

The present study aims to systematically investigate the effects of temperature, film thickness, and copolymer template on the thermal conductivity of ordered mesoporous nanocrystalline silicon thin films. First, sample film preparation and characterization were described. Then, the effective thermal conductivity measured using the $3 \omega$ method was reported from 25 to $315 \mathrm{~K}$. Finally, theoretical modeling was developed by combining the kinetic theory model and the coherent potential EMA. The model predictions were compared with experimental data.

\section{METHOD AND EXPERIMENTS}

3.1. Sample Film Preparation. The synthesis of ordered mesoporous silicon thin films similar to the ones investigated in the present study was previously described in detail in ref 26 .
Briefly, evaporation-induced self-assembly (EISA) using solgel silica precursors and a diblock copolymer template was used to produce the precursor material-an ordered mesoporous silica film. ${ }^{37}$ Mesoporous silicon was produced from the porous silica by magnesiothermic reduction. Here, two types of ordered mesoporous silica films were synthesized through EISA using two different diblock copolymers, namely, PB-PEO $[$ poly(butadiene)- $b$-poly(ethylene oxide) $]$ and PEP-PEO [poly(ethylene-propylene)- $b$-poly(ethylene oxide) $]$. Poly(butadiene(1,2 addition) )- $b$-poly(ethylene oxide), with a mass ratio of $\mathrm{PB}(5500)-b-\mathrm{PEO}$ (5000), a block ratio of $\mathrm{PB}_{102}-b-\mathrm{PEO}_{114}$, and with a polydispersity index $(\mathrm{PDI})=1.05$, was purchased from Polymer Source, Inc. Poly(ethylene-alt-propylene)- $b$-poly(ethylene oxide), with a mass ratio of PEP(3900)-b-PEO(4000), a block ratio of $\mathrm{PEP}_{56}-b-\mathrm{PEO}_{91}$, and with a PDI $=1.05$, was synthesized using reported methods. ${ }^{38,39}$ Briefly, polyisoprene was grown by anionic polymerization, terminated with an $-\mathrm{OH}$ group, and then hydrogenated over $\mathrm{Pd} / \mathrm{C}$. The resulting $\mathrm{PEP}-\mathrm{OH}$ was subsequently extended by anionic polymerization of ethylene oxide.

In the synthesis of the mesoporous silica, $30 \mathrm{mg}$ of diblock copolymer was dissolved in $2 \mathrm{~mL}$ of ethanol, and $600 \mathrm{mg}$ of TEOS and $300 \mathrm{mg}$ of $0.01 \mathrm{M} \mathrm{HCl}$ were mixed with $2 \mathrm{~mL}$ of ethanol in separate containers. These solutions were then mixed, and the resulting solution was stirred for $1 \mathrm{~h}$ and aged for 1 day. The films were dip-coated onto silicon substrates using a $5-10 \mathrm{~cm} / \mathrm{min}$ withdrawal rate in a chamber with $30 \%$ relative humidity. Films were dried overnight, heated at $60{ }^{\circ} \mathrm{C}$ for $24 \mathrm{~h}$, and then calcined at $450{ }^{\circ} \mathrm{C}$ using a $1{ }^{\circ} \mathrm{C} / \mathrm{min}$ heat ramp in air. The mesoporous silica films produced in this way had spheroidal pores $15 \mathrm{~nm}$ in diameter and an interpore distance of $\sim 25 \mathrm{~nm}$. To reduce silica into silicon, the mesoporous silica films were placed into a stainless steel chamber (inner volume $=5 \mathrm{~cm}^{3}$ ), and $5 \mathrm{mg}$ of $\mathrm{Mg}$ was located $\sim 1 \mathrm{~cm}$ away from the film. The chamber was then sealed in a glovebox with an argon atmosphere. The $\mathrm{Mg}$ vapor was generated by heating the chamber to $675{ }^{\circ} \mathrm{C}$ for $2 \mathrm{~h}$, followed by a $5 \mathrm{~h}$ soak at $675{ }^{\circ} \mathrm{C}$. Cooled films were immersed in $1 \mathrm{M}$ $\mathrm{HCl}$ for $10 \mathrm{~min}$ to remove the magnesia. This was followed by a $10 \mathrm{~min}$ immersion in $0.5 \% \mathrm{HF}$ to remove any residual silica. All chemistry performed on the silicon films was done under inert atmosphere.

3.2. Sample Characterization. Detailed characterization for each ordered mesoporous silicon film was performed using $\mathrm{X}$-ray diffraction (XRD) and scanning electron microscopy (SEM). Figure 1 shows low-angle XRD measurements for (a) PEP-PEO templated and (b) PB-PEO templated films at different stages of the synthesis process. It is evident that the mesostructure was preserved upon magnesium reduction and after both $\mathrm{HCl}$ and $\mathrm{HF}$ washes. However, some contraction was observed due to the thermal processing. The diffraction peaks are somewhat narrower for the PEP-PEO derived material, indicative of a more periodic nanometer scale architecture, but overall, both the PEP-PEO and PB-PEO copolymers resulted in relatively well-ordered cubic pore structure in the final mesoporous silicon.

Figure 2(a) shows an SEM image of the PEP-PEO templated silica film after calcination to remove the diblock copolymer template but before magnesium treatment. The high-quality spherical pore lattice is characteristic of simple oxide phases templated with these types of large diblock copolymer. Figures 2 (b) and 2(c) show top view images of mesoporous silicon films synthesized using PB-PEO and PEP-PEO, respectively, 

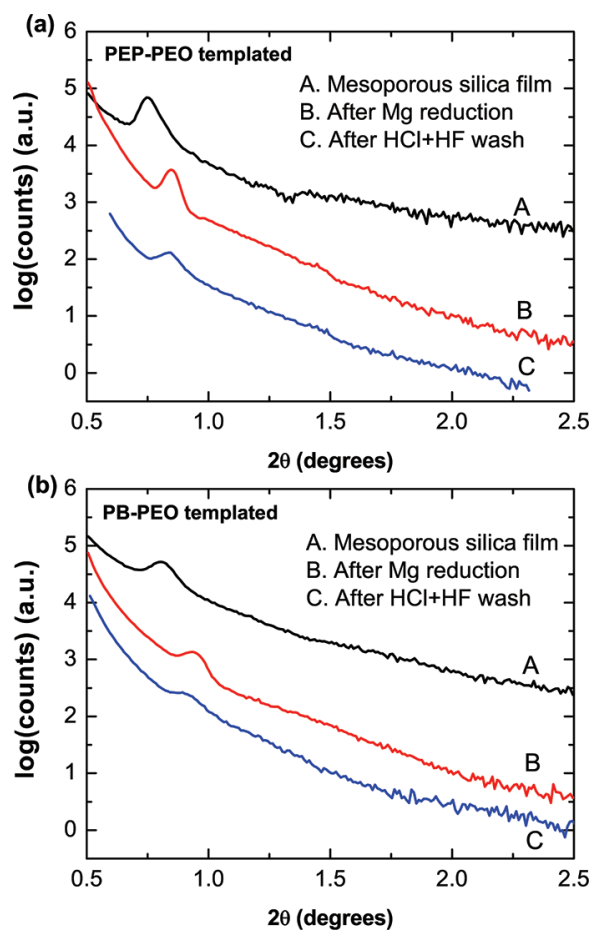

Figure 1. One-dimensional low-angle X-ray diffraction patterns for (a) PEP-PEO templated and (b) PB-PEO templated films including (A) mesoporous silica film before magnesium reduction (calcined at 450 ${ }^{\circ} \mathrm{C}$ in air), (B) mesoporous silicon film after $\mathrm{Mg}$ reduction treatment, and (C) mesoporous silicon film after $\mathrm{Mg}$ reduction and $\mathrm{HCl}$ and $\mathrm{HF}$ washes.

after reduction by magnesium and the subsequent $\mathrm{HCl}$ and $\mathrm{HF}$ washes. The images indicate that the PB-PEO and PEP-PEO templated silicon films have similar crystalline grain size. In addition, although the pores in the mesoporous silicon films shown in Figures 2(b) and 2(c) were not as well ordered or as regularly shaped as the pores shown in Figure 2(a), they retained the basic shape enforced by the template in the original silica framework. Overall, SEM images illustrate that the porosity does restructure somewhat upon $\mathrm{Mg}$ reduction, but they also clearly confirm that the periodicity produced by the diblock copolymer templates is preserved in the final material.

The film thickness was measured using cross-section SEM images with $\pm 15 \mathrm{~nm}$ uncertainty. Based on the top surface SEM images, the pore size, wall thickness, and crystal size were estimated to be about $16-18,13-15$, and $13 \mathrm{~nm}$, respectively. The porosity of $30 \% \pm 5 \%$ was expected to be similar to that measured in our previous study of KLE templated mesoporous silica. ${ }^{37}$ The $\pm 5 \%$ uncertainty accounted for the small change in porosity of the present samples due to the different copolymer templates and the crystallization of silicon during the reduction process. Table 1 summarizes the copolymer template, porosity, film thickness, wall thickness, crystal size, and pore diameter of the mesoporous silicon films investigated.

3.3. Thermal Conductivity Measurements. The crossplane thermal conductivity of ordered mesoporous silicon thin films was measured using the $3 \omega$ method with the so-called common-mode-subtraction technique. ${ }^{40,41}$ The principles, experimental apparatus, experimental procedure, and validation of the method have already been described elsewhere and need not be repeated. ${ }^{37,42}$ Briefly, a silicon nitride $\left(\mathrm{Si}_{x} \mathrm{~N}_{y}\right)$ layer,

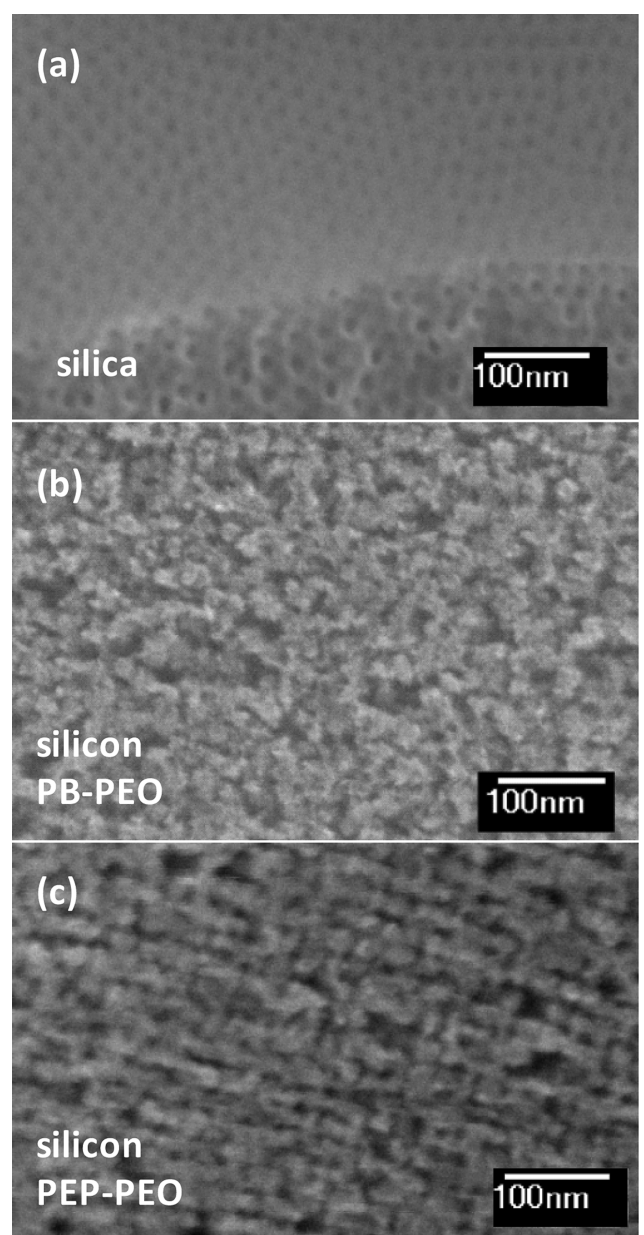

Figure 2. Top surface SEM images of (a) a PEP-PEO templated mesoporous silica film, (b) a PB-PEO templated silicon film after magnesium treatment and $\mathrm{HCl}$ and $\mathrm{HF}$ washes, and (c) a PEP-PEO templated silicon film after magnesium treatment and $\mathrm{HCl}$ and $\mathrm{HF}$ washes.

about $300 \mathrm{~nm}$ thick, was first deposited on the top surface of the mesoporous silicon films by plasma-enhanced chemical vapor deposition (PECVD). This silicon nitride film served both to seal and to protect the underlying mesoporous silicon film from oxidation and acted as an electrically insulating layer. An identical silicon nitride layer was also deposited on a bare silicon substrate in the same batch as the samples to serve as a reference. Then, metallic wires made of $10 \mathrm{~nm}$ thick $\mathrm{Cr}$ and 80 $\mathrm{nm}$ thick Au were deposited on both the sample and the reference using a standard lift-off process. The wires were 30 $\mu \mathrm{m}$ wide and $1 \mathrm{~mm}$ long and served as both heater and sensor. The samples were mounted inside a Janis ST100 cryostat, and the temperature was controlled between 25 and $315 \mathrm{~K}$ with $\pm 0.5 \mathrm{~K}$ uncertainty. A SR830 lock-in amplifier from Stanford Research Systems, Inc. was used to measure the third harmonic voltage response $V_{3 \omega}$ from the metallic wire.

The temperature oscillation amplitude $\Delta T$ was determined from the third harmonic voltage $V_{3 \omega}$ according to ${ }^{40}$

$$
\Delta T(\omega)=\frac{2 V_{3 \omega} R_{\mathrm{e}}}{V_{\omega}\left(\mathrm{d} R_{\mathrm{e}} / \mathrm{d} T\right)}
$$

where $V_{\omega}$ is the applied first harmonic AC voltage, while $\mathrm{d} R_{e} /$ $\mathrm{d} T$ is the derivative of the electrical resistance of the heater with respect to temperature. The electrical resistance $R_{\mathrm{e}}(T)$ was 
Table 1. Characteristics of the Mesoporous Silicon Thin Films Investigated

\begin{tabular}{|c|c|c|c|c|c|c|}
\hline sample no. & & porosity & thickness & wall thickness & crystal size & pore diameter \\
\hline \# & template & $f_{\mathrm{v}}(\%)$ & $t_{\mathrm{f}}(\mathrm{nm})$ & $t_{\mathrm{w}}(\mathrm{nm})$ & $d_{c}(\mathrm{~nm})$ & $d_{\mathrm{p}}(\mathrm{nm})$ \\
\hline 1 & PEP-PEO & $30 \pm 5$ & $140 \pm 15$ & $13-15$ & $\sim 13$ & $\sim 18$ \\
\hline 2 & PEP-PEO & $30 \pm 5$ & $175 \pm 15$ & $13-15$ & $\sim 13$ & $\sim 18$ \\
\hline 3 & PEP-PEO & $30 \pm 5$ & $260 \pm 15$ & $13-15$ & $\sim 13$ & $\sim 18$ \\
\hline 4 & PB-PEO & $30 \pm 5$ & $148 \pm 15$ & $13-15$ & $\sim 13$ & $\sim 16$ \\
\hline 5 & PB-PEO & $30 \pm 5$ & $235 \pm 15$ & $13-15$ & $\sim 13$ & $\sim 16$ \\
\hline 6 & PB-PEO & $30 \pm 5$ & $340 \pm 15$ & $13-15$ & $\sim 13$ & $\sim 16$ \\
\hline
\end{tabular}

measured before each $3 \omega$ measurement and was fitted with the Bloch-Grüneisen model. ${ }^{42,43}$ The amplitude of temperature oscillations in the mesoporous thin film $\Delta T_{\mathrm{f}}$ was then calculated by subtracting the amplitude of temperature oscillation of the deposited reference silicon nitride film $\Delta T_{\text {ref }}(\omega)$ from that of the mesoporous film with the protective silicon nitride film denoted by $\Delta T_{\text {tot }}(\omega)^{44}$

$$
\Delta T_{\mathrm{f}}=\Delta T_{\text {tot }}(\omega)-\Delta T_{\text {ref }}(\omega)
$$

where $\Delta T_{\mathrm{f}}$ was independent of frequency $\omega$. Finally, the thermal conductivity $k_{\mathrm{f}}$ of mesoporous silicon thin films was expressed as ${ }^{44}$

$$
k_{\mathrm{f}}=\frac{P t_{\mathrm{f}}}{2 b L \Delta T_{\mathrm{f}}}
$$

where $P$ is the electrical power dissipated in the metallic wire; $t_{\mathrm{f}}$ is the thickness of the mesoporous film; and $2 b$ and $L$ are the width and length of the gold heater, respectively. According to eqs 9-11, the total experimental uncertainty associated with $k_{\mathrm{f}}$ was typically between $6 \%$ and $15 \%$ for the films investigated and was mainly attributed to the uncertainty in $V_{3 \omega}( \pm 2 \%)$ and in $t_{\mathrm{f}}( \pm 15 \mathrm{~nm})$.

\section{MODELING}

In the present study, the effective medium approximation was combined with the kinetic theory model or the minimum thermal conductivity model described previously to predict the thermal conductivity of mesoporous silicon thin films.

First, the thermal conductivity of mesoporous silicon films was predicted using the kinetic theory model given by eq 7 where $k_{\mathrm{m}}$ was estimated using eqs $1-6$. The coefficients in the relaxation time models of eq 4 for Umklapp scattering $\tau_{\mathrm{U}, i}$ defect scattering $\tau_{\mathrm{D}, i}$, and boundary scattering $\tau_{\mathrm{B}, i}$ were determined as $B=1.25 \times 10^{-19} \mathrm{~s} / \mathrm{K}, C=157.2 \mathrm{~K}, D=3.21$ $\times 10^{-45} \mathrm{~s}^{3}$, and $E=0.0084 \mathrm{~m}$ by fitting eqs $1-4$ to the thermal conductivity of high purity single crystal silicon from 10 to $1400 \mathrm{~K}$ reported in ref 19 . In mesoporous silicon, phonon scattering by film boundaries was negligible compared with phonon scattering by grain boundaries since the thickness of the films investigated was at least 10 times larger than the crystallite grain size (Table 1). Thus, the total relaxation time for these films was given by eq 5 where the relaxation time model for phonon-grain boundary scattering $\tau_{\text {grain }, i}$ was predicted by eq $6 .^{23}$ The transmission parameter $\alpha$ was determined by fitting eqs $1-7$ to the thermal conductivity of mesoporous silicon films measured between 25 and $315 \mathrm{~K}$. It was found to be 0.093 and 0.051 for PEP-PEO and PB-PEO templated mesoporous silicon thin films, respectively. Both values were about 10 times smaller than those for dense (nonporous) nanocrystalline silicon reported by Wang et al. ${ }^{23}$ In fact, strong phonon scattering took place in mesoporous silicon at both intergrain and grain-pore boundaries. ${ }^{23,31}$ In the latter case, no phonon could transmit into the pores, i.e., $\alpha=0$. Overall, the averaged parameter $\alpha$ retrieved was greatly reduced compared with dense nanocrystalline silicon including those with high-energy grain boundaries. ${ }^{45}$

Finally, the minimum effective thermal conductivity of mesoporous silicon can be predicted by combining eqs 7 and 8 to yield

$$
k_{\text {eff,min }}=k_{\mathrm{m}, \min } \Psi_{\mathrm{cp}}\left(f_{\mathrm{v}}\right)=k_{\mathrm{m}, \min }\left(1-1.5 f_{\mathrm{v}}\right)
$$

where $k_{\mathrm{m}, \min }$ is the thermal conductivity of the strongly disordered matrix given by eq 8 . The term $\left(1-1.5 f_{\mathrm{v}}\right)$ accounts for the reduction in thermal conductivity due to the film mesoporosity.

\section{RESULTS AND DISCUSSION}

5.1. Dense Nanocrystalline Silicon. Figure 3 shows the thermal conductivity of the dense nanocrystalline silicon film

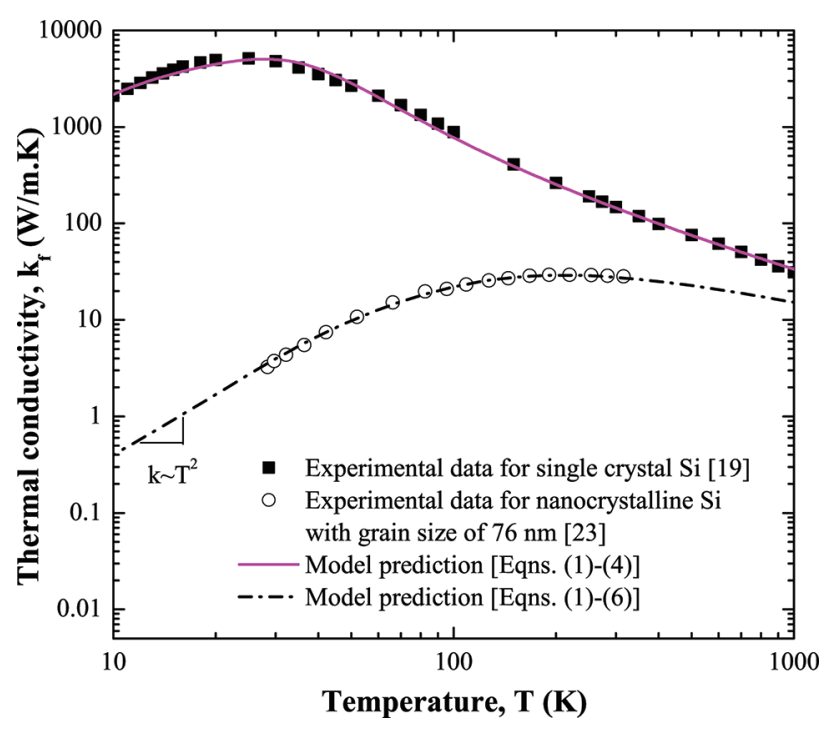

Figure 3. Thermal conductivity reported in the literature for high purity single crystal silicon ${ }^{19}$ and dense nanocrystalline silicon ${ }^{23}$ as a function of temperature. The solid and dashed lines correspond to the model predictions from the kinetic theory model [eqs 1-6] for single crystal and nanocrystalline silicon, respectively.

with grain size of $76 \mathrm{~nm}$ as a function of temperature reported by Wang et al. ${ }^{23}$ It also shows the experimental data for high purity single crystal silicon reported in ref 19 . The solid and dashed lines correspond to predictions from the kinetic theory model [eqs 1-6] for single crystal and nanocrystalline silicon, respectively. It is evident that the thermal conductivity of the dense nanocrystalline silicon film was between 1 and 4 orders of magnitude smaller than that of single crystal silicon, 
Table 2. Measured Thermal Conductivity of PEO-PEO and PB-PEO Templated Ordered Mesoporous Nanocrystalline Silicon Thin Films

\begin{tabular}{|c|c|c|c|c|c|c|}
\hline \multirow{2}{*}{$\frac{\text { temperature }}{(\mathrm{K})}$} & \multicolumn{3}{|c|}{ PEP-PEO templated samples } & \multicolumn{3}{|c|}{ PB-PEO templated samples } \\
\hline & sample 1 & sample 2 & sample 3 & sample 4 & sample 5 & sample 6 \\
\hline 25 & $0.022 \pm 0.002$ & $0.027 \pm 0.002$ & $0.028 \pm 0.002$ & $0.018 \pm 0.002$ & $0.014 \pm 0.001$ & $0.017 \pm 0.001$ \\
\hline 30 & $0.033 \pm 0.004$ & $0.040 \pm 0.004$ & $0.042 \pm 0.003$ & $0.025 \pm 0.003$ & $0.021 \pm 0.001$ & $0.024 \pm 0.001$ \\
\hline 36.5 & $0.049 \pm 0.006$ & $0.056 \pm 0.005$ & $0.061 \pm 0.003$ & $0.038 \pm 0.004$ & $0.030 \pm 0.002$ & $0.033 \pm 0.002$ \\
\hline 44.5 & $0.070 \pm 0.008$ & $0.076 \pm 0.007$ & $0.083 \pm 0.005$ & $0.050 \pm 0.005$ & $0.041 \pm 0.003$ & $0.045 \pm 0.002$ \\
\hline 54.2 & $0.093 \pm 0.010$ & $0.098 \pm 0.009$ & $0.11 \pm 0.01$ & $0.066 \pm 0.007$ & $0.054 \pm 0.004$ & $0.059 \pm 0.003$ \\
\hline 66 & $0.12 \pm 0.01$ & $0.12 \pm 0.01$ & $0.14 \pm 0.01$ & $0.084 \pm 0.009$ & $0.070 \pm 0.005$ & $0.074 \pm 0.004$ \\
\hline 80.4 & $0.15 \pm 0.02$ & $0.15 \pm 0.01$ & $0.17 \pm 0.01$ & $0.11 \pm 0.01$ & $0.088 \pm 0.006$ & $0.094 \pm 0.005$ \\
\hline 98 & $0.18 \pm 0.02$ & $0.18 \pm 0.02$ & $0.20 \pm 0.01$ & $0.13 \pm 0.01$ & $0.11 \pm 0.01$ & $0.12 \pm 0.01$ \\
\hline 119.3 & $0.21 \pm 0.02$ & $0.22 \pm 0.02$ & $0.24 \pm 0.02$ & $0.16 \pm 0.02$ & $0.13 \pm 0.01$ & $0.14 \pm 0.01$ \\
\hline 145.4 & $0.25 \pm 0.03$ & $0.25 \pm 0.02$ & $0.28 \pm 0.02$ & $0.18 \pm 0.02$ & $0.15 \pm 0.01$ & $0.16 \pm 0.01$ \\
\hline 177.1 & $0.27 \pm 0.03$ & $0.28 \pm 0.03$ & $0.31 \pm 0.02$ & $0.21 \pm 0.02$ & $0.18 \pm 0.01$ & $0.19 \pm 0.01$ \\
\hline 215.7 & $0.31 \pm 0.04$ & $0.31 \pm 0.03$ & $0.34 \pm 0.02$ & $0.23 \pm 0.03$ & $0.20 \pm 0.01$ & $0.21 \pm 0.01$ \\
\hline 262.7 & $0.33 \pm 0.04$ & $0.34 \pm 0.03$ & $0.37 \pm 0.03$ & $0.25 \pm 0.03$ & $0.22 \pm 0.02$ & $0.23 \pm 0.01$ \\
\hline 293 & $0.32 \pm 0.04$ & $0.34 \pm 0.03$ & $0.37 \pm 0.03$ & $0.26 \pm 0.03$ & $0.23 \pm 0.02$ & $0.25 \pm 0.01$ \\
\hline 315 & $0.32 \pm 0.04$ & $0.35 \pm 0.03$ & $0.37 \pm 0.03$ & $0.25 \pm 0.03$ & $0.23 \pm 0.02$ & $0.25 \pm 0.01$ \\
\hline
\end{tabular}

depending on the temperature range examined. This can be attributed to strong phonon scattering by grain boundaries. Importantly, the model predictions agreed very well with experimental data for both single crystal silicon and dense nanocrystalline silicon, validating both of these models.

Moreover, Figure 3 shows that the thermal conductivity of bulk single crystal silicon decreases with increasing temperature for temperatures above $30 \mathrm{~K}$. This is due to phonon Umklapp scattering which dominated phonon scattering beyond $30 \mathrm{~K}^{27}$ On the other hand, the thermal conductivity of dense nanocrystalline silicon did not reach a maximum until about $200 \mathrm{~K}$, after which it started to decrease with increasing temperature. This can be explained by the fact that phonon scattering by crystallite grain boundaries dominated at low temperatures, while Umklapp scattering became important beyond $200 \mathrm{~K}$. The measured thermal conductivity and the model predictions for the nanocrystalline silicon were found to be proportional to $T^{2}$ at low temperatures $(T<50 \mathrm{~K})$. This is also in good agreement with data reported by Wang et al. ${ }^{23}$ Note that the temperature dependence of $k \propto T^{2}$ is characteristic of amorphous or strongly disordered materials at low temperatures. ${ }^{27}$ For single crystalline silicon, $k$ is proportional to $T^{3}$ below $10 \mathrm{~K}^{19}$

5.2. Mesoporous Nanocrystalline Silicon. Table 2 summarizes the thermal conductivity of PEO-PEO and PBPEO templated mesoporous nanocrystalline silicon thin films measured at different temperatures. Figure 4 shows the measured thermal conductivity of all PB-PEO and PEP-PEO templated ordered mesoporous nanocrystalline silicon thin films as a function of temperature. It also shows the predictions from coherent potential approximation combined with the kinetic theory model [eqs 1-7] or the minimum effective thermal conductivity model [eq 12 ]. Figure 4 establishes that the measured thermal conductivity of mesoporous silicon thin films varied from 0.01 to $0.4 \mathrm{~W} / \mathrm{m} \cdot \mathrm{K}$ as temperature increased from 25 to $315 \mathrm{~K}$. The thermal conductivity was between 3 and 5 orders of magnitude smaller than that of single crystal silicon over the same temperature range. The reduction was mainly attributed to the presence of pores and strong phonon scattering by crystallite grain boundaries. Interestingly, the measured thermal conductivity of these mesoporous silicon films was about 100 times less than that reported for dense

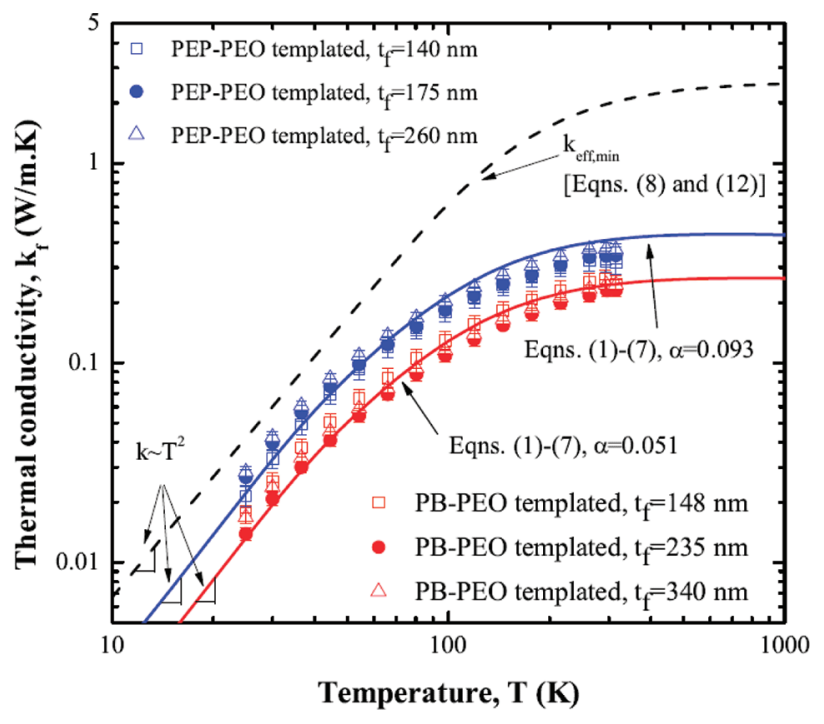

Figure 4. Measured thermal conductivity of mesoporous silicon thin films as a function of temperature from 25 to $315 \mathrm{~K}$. The lines correspond to the model predictions from the kinetic theory model [eqs 1-7] and the effective minimum thermal conductivity model [eqs 8 and 12].

nanocrystalline silicon by Wang et al. ${ }^{23}$ This was due to the facts that (i) the crystallite size (13 nm) was smaller and (ii) the silicon films measured in the present study were mesoporous with porosity of about $30 \%$. Moreover, it is interesting to note that the room-temperature thermal conductivity of mesoporous silicon films ranged from 0.23 to $0.37 \mathrm{~W} / \mathrm{m} \cdot \mathrm{K}$. It was as low as that of mesoporous amorphous silica films ${ }^{37}$ despite the crystallinity of the mesoporous Si films.

Comparison between PEP-PEO and PB-PEO templated films indicated that the thermal conductivity of the PB-PEO templated mesoporous silicon thin films was about $30-40 \%$ smaller than that of the PEP-PEO templated films over the entire temperature range. This can be attributed to the fact that the PB-PEO templated films had smaller pore diameter than the PEP-PEO templated films (Table 1). For a given porosity, a smaller pore resulted in larger pore surface area per unit volume which increased phonon scattering by pore boundaries. ${ }^{31}$ The 
increased disorder in the PB-PEO templated films may also have contributed to the reduced thermal conductivity.

The differences in thermal conductivity between the PEPPEO and PB-PEO templated films were interesting because the films were generally very similar and differed only in the details of the nanometer scale morphology. That morphology stemmed from the structure of the initial mesoporous silica films. It can be tuned over a fairly broad range using different polymer templates and/or concentrations. ${ }^{37}$ This results in tunable porosity, pore size, pore arrangement, and wall thickness. Because the structure of the mesoporous silica films is mostly retained during magnesium reduction, this synthetic method gives one the ability to tune the structure of the templated mesoporous nanocrystalline silicon thin films to achieve the desired thermal conductivity.

Moreover, examination of films of different thickness indicates that the film thickness had a negligible effect on the measured thermal conductivity for both PEP-PEO and PB-PEO templated thin films, considering the experimental uncertainty. Instead, phonon scattering by crystallite grain boundaries dominated over phonon scattering by film boundaries. The slight difference in thermal conductivity for each type of mesoporous silicon thin films could be attributed to small variations in morphology from one sample to another, including porosity, crystallite size, and wall thickness.

Figure 4 also establishes that the thermal conductivity predictions from the kinetic theory model, with fitting parameter $\alpha=0.093$ for PEP-PEO templated films and $\alpha=$ 0.051 for PB-PEO templated films, captured the behavior of the measured data over the entire temperature range. In addition, the thermal conductivity of mesoporous silicon films increased monotonically with increasing temperature and reached a plateau beyond $300 \mathrm{~K}$. This could be explained by the facts that (i) more phonon modes were excited as temperature increased and contributed to heat transfer and (ii) their contribution was compensated by the simultaneous increase in the phonon Umklapp scattering rate beyond $300 \mathrm{~K}$. Moreover, at low temperatures $(T<50 \mathrm{~K})$, both measured data and model predictions were found to be proportional to $T^{2}$, in accordance with that observed for dense nanocrystalline silicon. ${ }^{23}$ Indeed, mesoporous nanocrystalline silicon has a strongly disordered nanostructure, similar to the dense nanocrystalline silicon, and thus, it features amorphous-like thermal conductivity at low temperatures.

Finally, it is interesting to note that the predictions from the minimum effective thermal conductivity model [eq 12 ] also varied linearly with $T^{2}$ at low temperatures. However, this model failed to predict the temperature dependence of the measured data at high temperatures. This can be attributed to the fact that, at high temperatures, numerous phonon modes contributed to heat transfer, and Umklapp scattering became important, resulting in more crystalline-like thermal conductivity which was not captured by the minimum thermal conductivity model.

\section{CONCLUSION}

This paper reports preparation, characterization, and crossplane thermal conductivity measurements for ordered mesoporous nanocrystalline silicon thin films from 25 to $315 \mathrm{~K}$. Overall, the measured thermal conductivity was between 3 and 5 orders of magnitude smaller than that of bulk single crystal silicon, depending on the temperature range examined. In addition, thin films templated by PB-PEO copolymer had smaller thermal conductivity than those templated by PEP-PEO copolymer due to their smaller pores and increased disorder. The mesoporous silicon thin films had amorphous-like thermal conductivity proportional to $T^{2}$ at low temperatures. On the other hand, they had crystalline-like thermal conductivity at high temperatures as it reached a maximum and plateau beyond $300 \mathrm{~K}$. Finally, a kinetic theory model was used to predict the effective thermal conductivity of mesoporous silicon thin films accounting for (i) phonon scattering by defects and crystallite grain boundaries as well as due to Umklapp scattering in the nanocrystalline matrix and (ii) the porosity of the films. Good agreement was found between the measured data and the model predictions. These results and the model could help establish new strategies to control the thermal conductivity of mesoporous silicon for a wide range of applications including thermoelectric energy conversion.

\section{AUTHOR INFORMATION}

\section{Corresponding Author}

*Phone: +1 (310)-206-5598. Fax: +1 (310)-206-4830. E-mail: pilon@seas.ucla.edu; tolbert@chem.ucla.edu.

\section{Notes}

The authors declare no competing financial interest.

\section{ACKNOWLEDGMENTS}

We gratefully acknowledge the contributions of Dr. Morgan Stefik and Prof. Ulrich Wiesner (Cornell University, Materials Science and Engineering) for synthesizing the PEP-PEO diblock copolymer used in this work. This work was supported by the Petroleum Research Fund of the American Chemical Society under Grant ACS PRF\# 46107-AC5, by the National Science Foundation under Grant CHE-1112569, and by the Center for Molecularly Engineered Energy Materials (MEEM), an Energy Frontier Research Center funded by the U.S. Department of Energy (D.O.E.), Office of Science, Office of Basic Energy Sciences under Award Number DE-SC0001342.

\section{REFERENCES}

(1) Cullis, A. G.; Canham, L. T.; Calcott, P. D. J. J. Appl. Phys. 1997, 82 (3), 909-965.

(2) Roussel, P.; Lysenko, V.; Remaki, B.; Delhomme, G.; Dittmar, A.; Barbier, D. Sens. Actuators, A 1999, 74 (1-3), 100-103.

(3) Mikulec, F. V.; Kirtland, J. D.; Sailor, M. J. Adv. Mater. 2002, 14 (1), 38-41.

(4) Plessis, M. d. Sens. Actuators, A 2007, 135 (2), 666-674.

(5) Currano, L. J.; Churaman, W. A. J. MEMS 2009, 18 (4), 799807.

(6) Lee, J.-H.; Grossman, J. C.; Reed, J.; Galli, G. A. Appl. Phys. Lett. 2007, 91 (22), 223110.

(7) Lee, J.-H.; Galli, G. A.; Grossman, J. C. Nano Lett. 2008, 8 (11), 3750-3754.

(8) Bux, S. K.; Blair, R. G.; Gogna, P. K.; Lee, H.; Chen, G.; Dresselhaus, M. S.; Kaner, R. B.; Fleurial, J.-P. Adv. Funct. Mater. 2009, 19 (15), 2445-2452.

(9) Drost, A.; Steiner, P.; Moser, H.; Lang, W. Sensor Mater. 1995, 7 (2), 111-120.

(10) Benedetto, G.; Boarino, L.; Spagnolo, R. Appl. Phys. A: Mater. Sci. Process. 1997, 64 (2), 155-159.

(11) Bernini, U.; Maddalena, P.; Massera, E.; Ramaglia, A. J. Opt. A: Pure Appl. Opt. 1999, 1 (2), 210-213.

(12) Amato, G.; Angelucci, R.; Benedetto, G.; Boarino, L.; Dori, L.; Maccagnani, P.; Rossi, A.; Spagnolo, R. J. Porous Mater. 2000, 7 (1-3), 183-186.

(13) Shen, Q.; Toyoda, T. Rev. Sci. Instrum. 2003, 74 (1), 601-603. 
(14) Périchon, S.; Lysenko, V.; Remaki, B.; Barbier, D. J. Appl. Phys. 1999, 86 (8), 4700-4702.

(15) Wolf, A.; Brendel, R. Thin Solid Films 2006, 513 (1-2), 385390.

(16) Gomès, S.; David, L.; Lysenko, V.; Descamps, A.; Nychyporuk, T.; Raynaud, M. J. Phys. D: Appl. Phys. 2007, 40 (21), 6677-6683.

(17) Hammiche, A.; Pollock, H. M.; Song, M.; Hourston, D. J. Meas. Sci. Technol. 1996, 7 (2), 142-150.

(18) Callard, S.; Tallarida, G.; Borghesi, A.; Zanotti, L. J. Non-Cryst. Solid 1999, 245 (1-3), 203-209.

(19) Touloukian, Y. S.; Powell, R. W.; Ho, C. Y.; Klemens, P. G. Thermophysical Properties of Matter-Thermal Conductivity of Metallic Elements and Alloys; TPRC Data Series; IFI/Plenum: New York, NY, 1970; Vol. 1.

(20) Gesele, G.; Linsmeier, J.; Drach, J.; Fricke, J.; Aren-Fischer, R. J. Phys. D: Appl. Phys. 1997, 30 (21), 2911-2916.

(21) Song, D.; Chen, G. Appl. Phys. Lett. 2004, 84 (5), 687-689.

(22) Hopkins, P. E.; Reinke, C. M.; Su, M. F.; Olsson, R. H.; Shaner, E. A.; Leseman, Z. C.; Serrano, J. R.; Phinney, L. M.; El-Kady, I. Nano Lett. 2011, 11 (1), 107-112.

(23) Wang, Z.; Alaniz, J. E.; Jang, W.; Garay, J. E.; Dames, C. Nano Lett. 2011, 11 (6), 2206-2213.

(24) Lehmann, V.; Stengl, R.; Luigart, A. Mater. Sci. Eng., B 2000, 69-70 (14), 11-22.

(25) Tan, K. W.; Saba, S. A.; Arora, H.; Thompson, M. O.; Wiesner, U. ACS Nano 2011, 5 (10), 7960-7966.

(26) Richman, E. K.; Kang, C. B.; Brezesinski, T.; Tolbert, S. H. Nano Lett. 2008, 8 (9), 3075-3079.

(27) Tien, C. L.; Majumdar, A.; Gerner, F. M. Microscale Energy Transport; Taylor and Francis: Washington, DC, 1998.

(28) Hopkins, P. E.; Rakich, P. T.; Olsson, R. H.; El-kady, I. F.; Phinney, L. M. Appl. Phys. Lett. 2009, 95 (16), 161902.

(29) Nilsson, G.; Nelin, G. Phys. Rev. B 1972, 6 (10), 3777-3786.

(30) Mingo, N. Phys. Rev. B 2003, 68 (11), 113308.

(31) Fang, J.; Pilon, L. J. Appl. Phys. 2011, 110 (6), 064305.

(32) Landauer, R. J. Appl. Phys. 1952, 23 (7), 779-784.

(33) Cahill, D. G.; Allen, T. H. Appl. Phys. Lett. 1994, 65 (3), 309311.

(34) Coquil, T.; Fang, J.; Pilon, L. Int. J. Heat Mass Transfer 2011, 54 (21-22), 4540-4548.

(35) Fang, J.; Pilon, L. In Proceedings of the ASME 2012 3rd Micro/ Nanoscale Heat \& Mass Transfer Conference, MNHMT2012-75153, Atlanta, Georgia, USA, March 3-6, 2012.

(36) Cahill, D. G.; Pohl, R. O. Annu. Rev. Phys. Chem. 1988, 39, 93121.

(37) Coquil, T.; Richman, E. K.; Hutchinson, N.; Tolbert, S. H.; Pilon, L. J. Appl. Phys. 2009, 106 (3), 034910.

(38) Hillmyer, M. A.; Bates, F. S. Macromolecules 1996, 29 (22), 6994-7002.

(39) Allgaier, J.; Poppe, A.; Willner, L.; Richter, D. Macromolecules 1997, 30 (6), 1582-1586.

(40) Cahill, D. G. Rev. Sci. Instrum. 1990, 61, 802-808.

(41) Kimling, J.; Martens, S.; Nielsch, K. Rev. Sci. Instrum. 2011, 82 (7), 074903

(42) Fang, J.; Reitz, C.; Brezesinski, T.; Nemanick, E. J.; Tolbert, S. H.; Pilon, L. J. Phys. Chem. C 2011, 115 (30), 14606-14614.

(43) Ziman, J. M. Electron and Phonons; Oxford University Press: Oxford, UK, 1960.

(44) Cahill, D. G.; Katiyar, M.; Abelson, J. R. Phys. Rev. B 1994, 50 (9), 6077-6081.

(45) Ponomareva, I.; Srivastava, D.; Menon, M. Nano Lett. 2007, 7 (5), 1155-1159. 